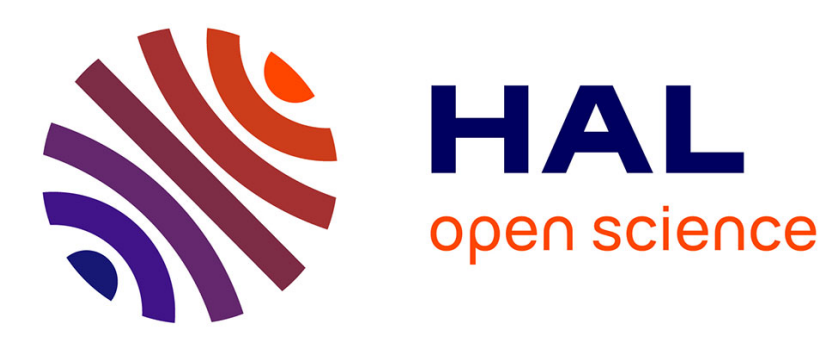

\title{
The deep atmosphere of Venus and the possible role of density-driven separation of $\mathrm{CO} 2$ and $\mathrm{N} 2$
}

Sébastien Lebonnois, Gerald Schubert

\section{To cite this version:}

Sébastien Lebonnois, Gerald Schubert. The deep atmosphere of Venus and the possible role of densitydriven separation of CO2 and N2. Nature Geoscience, 2017, 10 (7), pp.473 - 477. 10.1038/ngeo2971. hal-01635402

\section{HAL Id: hal-01635402 \\ https://hal.science/hal-01635402}

Submitted on 15 Nov 2017

HAL is a multi-disciplinary open access archive for the deposit and dissemination of scientific research documents, whether they are published or not. The documents may come from teaching and research institutions in France or abroad, or from public or private research centers.
L'archive ouverte pluridisciplinaire HAL, est destinée au dépôt et à la diffusion de documents scientifiques de niveau recherche, publiés ou non, émanant des établissements d'enseignement et de recherche français ou étrangers, des laboratoires publics ou privés. 


\title{
The deep atmosphere of Venus and the possible role of density-driven separation of $\mathrm{CO}_{2}$ and $\mathrm{N}_{2}$
}

\author{
Sebastien Lebonnois, ${ }^{1 *}$ Gerald Schubert ${ }^{2}$ \\ ${ }^{1}$ Laboratoire de Météorologie Dynamique (LMD/IPSL), \\ Sorbonne Universités, UPMC Univ Paris 06, ENS, PSL Research University, \\ Ecole Polytechnique, Université Paris Saclay, CNRS, Paris, France \\ ${ }^{2}$ Department of Earth, Planet. and Space Sci., UCLA, CA, USA \\ *e-mail: sebastien.lebonnois@1md.jussieu.fr. \\ Sorbonne Universités, UPMC Univ Paris 06, ENS, PSL Research University,
}

\begin{abstract}
With temperatures around $700 \mathrm{~K}$ and pressures of around $75 \mathrm{bar}$, the deepest
\end{abstract} 12 kilometres of the atmosphere of Venus are so hot and dense that the atmosphere behaves like a supercritical fluid. The Soviet VeGa-2 probe descended through the atmosphere in 1985 and obtained the only reliable temperature profile for the deep Venusian atmosphere thus far. In this temperature profile, the atmosphere appears to be highly unstable at altitudes below $7 \mathrm{~km}$, contrary to expectations. We argue that the VeGa-2 temperature profile could be explained by a change in the atmospheric gas composition, and thus molecular mass, with depth. We propose that the deep atmosphere consists of a non-homogeneous layer in which the abundance of $\mathbf{N}_{2}$ - the second most abundant constituent of the Venusian atmosphere after $\mathrm{CO}_{2}$ - gradually decreases to near-zero at the surface. It is difficult to explain a decline in $\mathbf{N}_{2}$ towards the surface with known nitrogen sources and sinks for Venus. Instead we suggest, 
partly based on experiments on supercritical fluids, that density-driven separation of $\mathrm{N}_{2}$ from $\mathrm{CO}_{2}$ can occur under the high pressures of Venus's deep atmosphere, possibly by molecular diffusion, or by natural density-driven convection. If so, the amount of nitrogen in the atmosphere of Venus is $15 \%$ lower than commonly assumed. We suggest that similar density-driven separation could occur in other massive planetary atmospheres.

Venus has a massive and scorching atmosphere. With a surface pressure of 92 bars its atmosphere is 92 times as massive as Earth's atmosphere. At the surface of Venus the temperature is $464^{\circ} \mathrm{C}$, hot enough to melt lead. Atmospheric density at the surface is about $65 \mathrm{~kg} \mathrm{~m}^{-3}$ or $6.5 \%$ the density of liquid water. ${ }^{1}$ Atmospheric composition is $96.5 \% \mathrm{CO}_{2}$ and $3.5 \% \mathrm{~N}_{2}$ (by volume). ${ }^{2}$ Minor gases include $\mathrm{SO}_{2}, \mathrm{Ar}, \mathrm{H}_{2} \mathrm{O}$, and $\mathrm{CO} .{ }^{3,4} \mathrm{SO}_{2}$ at the level of only $150 \mathrm{ppm}$ is particularly important because of the blanket of sulfuric acid clouds that completely shroud the planet from view. ${ }^{5}$ The clouds effectively reflect the solar radiation incident on Venus resulting in a bond albedo of 0.77 , more than double that of the Earth at 0.31 . As a consequence more sunlight is absorbed at the surface of Earth than at Venus' surface even though Venus is $72 \%$ nearer to the Sun. The temperature distribution in Venus' atmosphere is determined in large part by its absorption of sunlight. ${ }^{1}$ Temperature and pressure are so large at Venus' surface that the atmosphere is a supercritical fluid.

In addition to the basic properties above we have detailed knowledge of the atmospheric structure (altitude profiles of temperature and pressure and locations of the clouds) from decades of observation by orbiting spacecraft (Soviet Venera 15 and $16,{ }^{6-8}$ U.S. Pioneer Venus Orbiter $^{9,10}$ and Magellan, ${ }^{11}$ ESA Venus-Express ${ }^{12-14}$ and the ongoing Japanese Akatsuki), entry probes and landers, ${ }^{15-18}$ balloons, ${ }^{17}$ and Earth-based telescopes ${ }^{3,19-21}$ (Fig. 1). These observations have shown that Venus, like Earth, has a troposphere extending from the surface to the upper cloud region at about 60 to $65 \mathrm{~km}$ altitude, wherein temperature decreases with height. ${ }^{1,22}$ 
The sulfuric acid clouds extend downward to about $48 \mathrm{~km}$ altitude. ${ }^{5}$ Above the clouds are regions of the atmosphere analogous to Earth's mesosphere and thermosphere but our focus here is the atmosphere below the clouds. At cloud heights atmospheric temperature and pressure are similar to those at the Earth's surface. There is no stratosphere on Venus similar to Earth's stratosphere that is heated by ozone absorption of solar ultraviolet radiation.

The altitude profile of temperature allows identification of stable layers and layers of convective activity. There is a convective region in the clouds between about 50 and $55 \mathrm{~km}$ altitude, ${ }^{14,23}$ as experienced by the Soviet VeGa-1 and VeGa-2 balloons that cruised in this layer. ${ }^{17}$ Below this region extending downward to about $32 \mathrm{~km}$ altitude the atmosphere is stable. Below this stable layer the atmosphere is well mixed down to an altitude of about $18 \mathrm{~km}$. At even greater depth, the atmosphere is stable again until an altitude of about $7 \mathrm{~km}$. The nature of the lowest $7 \mathrm{~km}$ of the atmosphere, a layer that contains $37 \%$ of the mass of the atmosphere, is at the heart of our discussion.

While the exploration of Venus' atmosphere has been extensive, as discussed above, the deep atmosphere remains a largely unobserved region. It is challenging to obtain data remotely below the thick cloud layer covering the planet. Many probes have been sent to the surface of Venus: the Soviet Venera mission series, ${ }^{15}$ the U.S. Pioneer Venus probes,${ }^{16}$ and the Soviet VeGa probes. ${ }^{17,18}$ These probes measured temperature $(T)$ and pressure $(p)$ during descent, and made measurements of atmospheric composition, showing that the two major constituents were carbon dioxide $\left(\mathrm{CO}_{2}, 96.5 \%\right)$ and nitrogen $\left(\mathrm{N}_{2}, 3.5 \%\right)^{2,24,25}$ Unfortunately, almost no temperature data were obtained from the deepest layers of Venus' atmosphere, since most Venera probe temperature profiles had large uncertainties and all the Pioneer Venus probe temperature experiments stopped functioning at $12 \mathrm{~km}$ above the surface. ${ }^{22}$ The Pioneer Venus temperature profiles below $12 \mathrm{~km}$ were reconstructed from pressure measurements, extrapolation of $T(p)$ and iterative altitude computation, ${ }^{16}$ and only these reconstructions (prone to significant 
uncertainties) and the Venera 10 profile $^{26}$ were used to build the Venus International Reference Atmosphere model. ${ }^{22}$ The only available and reliable temperature profile reaching to the surface was acquired by the VeGa-2 probe ${ }^{17,18,27}$ (Fig. 2). Measurements were done with two different platinum wires (one bare, one protected in a thin ceramic shield), with a measured accuracy of $\pm 0.5 \mathrm{~K}$ from 200 to $800 \mathrm{~K}$. The time constants of the two detectors were $0.1 \mathrm{~s}$ and $3 \mathrm{~s}$. The delay of the second detector induced systematic shift between the two measurements, with differences no larger than $2 \mathrm{~K}$ down to the surface. ${ }^{17}$ The measured temperature profile fits remarkably well with the Pioneer Venus and VIRA profiles above roughly $15 \mathrm{~km}$ altitude. ${ }^{27}$ This illustrates the small temporal and spatial variability of the temperature in the deep atmosphere of Venus, with differences between the different observed profiles smaller than $5 \mathrm{~K}$ (and not depending on altitude).

Below $7 \mathrm{~km}$, a region where no precise measurements of $\mathrm{N}_{2}$ abundance was published, ${ }^{2}$ the VeGa-2 temperature profile showed a strongly unstable vertical temperature gradient that has remained unexplained since VeGa-2 landed on Venus on June 15, 1985. ${ }^{27,28}$ The difference in temperature between the adiabatic profile (neutral stability) and the observed profile is up to roughly $9 \mathrm{~K}$ around $7 \mathrm{~km}$. This interface region between the surface and the atmosphere, called the planetary boundary layer (PBL), controls how the angular momentum and energy are exchanged between the two reservoirs. Characterization of the mixing processes occuring in the PBL is crucial to understanding the angular momentum budgets of the atmosphere and solid planet. This is particularly true in the case of Venus, which is characterized by a peculiar atmospheric circulation, the superrotation: the whole atmosphere is rotating much faster than the surface below, with maximum zonal winds reaching more than $100 \mathrm{~m} / \mathrm{s}$ at the altitude of the cloud top $(70 \mathrm{~km}) .{ }^{29}$ This large zonal rotation of the massive Venus atmosphere makes its atmospheric angular momentum a relatively large fraction $\left(1.6 \times 10^{-3}\right)$ of the angular momentum of the solid body. For Earth this fraction is $2.7 \times 10^{-8}$. Exchanges of angular momentum between 
the two reservoirs would lead to changes in the length of day of Venus and zonal wind speeds in the atmosphere.

A possible interpretation of this peculiar temperature structure involves unexpected properties of the $\mathrm{CO}_{2} / \mathrm{N}_{2}$ mixture in high-pressure, high-temperature conditions, which are not well known. This is illustrated by a recent experiment that shows a vertical separation between these two compounds within the fluid phase, a behavior difficult to explain. ${ }^{30}$ Despite a lack of theoretical and experimental constraints, this density-driven separation may be the key to understanding the structure of the deepest layers of Venus' atmosphere.

\section{Stability in the deep atmosphere of Venus}

The temperature profile close to the surface is a very good indicator of the properties of the PBL. In addition to the static stability, the potential temperature is an efficient variable to analyze the stratification of the atmosphere (Box 1). The vertical profiles of the potential temperature derived from the VeGa-2 and Pioneer Venus probes are displayed in Fig. 3. Layers with constant potential temperature are layers where the temperature follows the adiabatic lapse rate, indicative of convection or large-scale vertical mixing. Below roughly $7 \mathrm{~km}$, the vertical gradient of the VeGa-2 potential temperature is approximately constant and strongly negative $(-1.5 \mathrm{~K} / \mathrm{km})$, corresponding to a highly unstable situation. Such a profile of potential temperature is never observed on Earth. On Mars, radiative surface heating sometimes drives a very unstable surface layer, yielding highly active convection up to $9 \mathrm{~km}$ above surface. In these conditions, the potential temperature may display negative gradients over the surface, up to 1 or $2 \mathrm{~km}$ altitude. ${ }^{31}$ For Venus, this situation is unlikely, as direct heating of the surface is only a small fraction of that of Mars' surface. ${ }^{32}$

However, the VeGa-2 probe potential temperature profile can be understood if the stability of this layer is altered by a vertical gradient in the mean molecular mass $(\mu)$, i.e., in the atmospheric 
gas composition (as detailed in the online Methods section): the assumption that this layer is close to convective instability yields a vertical profile of mean molecular mass which is almost linear with the logarithm of pressure, from $43.44 \mathrm{~g} / \mathrm{mol}$ above $7 \mathrm{~km}$ to $44.0 \mathrm{~g} / \mathrm{mol}$ at the surface.

\section{A density-driven gas separation hypothesis}

Though a systematic error in the temperature measurements can not be excluded, the fact that this error would have maintained a stable vertical temperature gradient from $7 \mathrm{~km}$ altitude to the surface, for both VeGa-2 temperature sensors is unlikely. If this temperature profile is accurate, then it may be neutrally stable with the previously mentioned variation in the mean molecular mass $\mu$. The value obtained in this case for $\mu$ at the surface is remarkably close to that of pure $\mathrm{CO}_{2}$, so that an intriguing, but very simple explanation for the vertical profile of $\mu$ is a regular decrease in $\mathrm{N}_{2}$ mole fraction, from $3.5 \%$ above $7 \mathrm{~km}$ to almost zero at the surface. Such a composition variation would have a significant impact on the total amount of nitrogen contained in the atmosphere, which would decrease to only $85 \%$ of the total amount for a well mixed atmosphere. This could have potential implications for studies that investigate the respective nitrogen inventories of Earth and Venus. ${ }^{33}$ The increase of the mean molecular mass towards the surface might also be consistent with an increase in the abundance of an atmospheric compound heavier than $\mathrm{CO}_{2}$, though this would be an even more puzzling coincidence. For an increase up to the $0.1 \%$ level at the surface, the molar mass of the component would need to be of the order of $560 \mathrm{~g} / \mathrm{mol}$. A lower molar mass would mean a higher abundance. Solutions could be found, but it seems quite unlikely that the change of composition would be different from the decrease of $\mathrm{N}_{2}$ abundance as the surface is approached.

Based on this hypothetical interpretation of the VeGa-2 probe temperature profile, the gradient in $\mathrm{N}_{2}$ abundance obtained in Venus's deep atmosphere is around $5 \mathrm{ppm} / \mathrm{m}$. In planetary atmospheres, such vertical gradients of composition are usually associated with sources or sinks 
of the varying compound, such as chemistry, condensation, or surface processes. However, the hypothesis that this nitrogen gradient might be the result of a surface sink faces serious difficulties. It would require a constant downward flux of nitrogen, that would need to be sustained over geological times unless a recycling process or an equivalent source could drive nitrogen back into the atmosphere.

Another possibility is explored here : this gradient may result from an equilibrium state due to separation of nitrogen from carbon dioxide in the dense conditions of Venus's deep atmosphere. Such a separation of $\mathrm{N}_{2}$ and $\mathrm{CO}_{2}$ in high-pressure conditions is illustrated by recent experiments. ${ }^{30,34}$ Though the conditions of these experiments are clearly different from conditions in the deep atmosphere of Venus, it demonstrates the impact of high densities on the $\mathrm{CO}_{2} / \mathrm{N}_{2}$ binary mixture. In the first of these experiments, ${ }^{30}$ a mixture of $50 \% \mathrm{~N}_{2} / 50 \% \mathrm{CO}_{2}$ (mole fractions) was put in an 18-cm high vessel at room temperature for pressures above 100 bars. At $p=100$ bars and $T=23^{\circ} \mathrm{C}$, the $\mathrm{CO}_{2} / \mathrm{N}_{2}$ mixture is supercritical, not far above the critical point of the fluid mixture $\left(T_{C}=-9.3^{\circ} \mathrm{C}, p_{C}=98\right.$ bar $)$, and $\mathrm{CO}_{2}$ departs slightly from being ideal. Using the equations of state for pure $\mathrm{CO}_{2}$ and $\mathrm{N}_{2},{ }^{34,35} \mathrm{CO}_{2}$ partial pressure is 44 bars, $\mathrm{CO}_{2}$ density is $101 \mathrm{~kg} / \mathrm{m}^{3}$ and total density in the vessel is around $165 \mathrm{~kg} / \mathrm{m}^{3}$, to be compared with the densities in the deep Venusian atmosphere: 40 to $70 \mathrm{~kg} / \mathrm{m}^{3}$ for pressures higher than 50 bars. In these experimental conditions, $\mathrm{N}_{2}$ and $\mathrm{CO}_{2}$ were observed to separate significantly along the vertical dimension, $\mathrm{N}_{2}$ reaching over $70 \%$ mole fraction at the top of the vessel, while $\mathrm{CO}_{2}$ reached almost $90 \%$ at the bottom. ${ }^{30}$ Over the $18 \mathrm{~cm}$ of the experimental vessel, this separation is extreme, with an average gradient of 3 to $4 \% / \mathrm{cm}$. In Venus's deep atmosphere, the $5 \mathrm{ppm} / \mathrm{m}$ gradient in $\mathrm{N}_{2}$ abundance appears much smaller in comparison.

The molecular diffusion in this binary gas mixture includes three terms: one due to the compositional gradient, one due to the temperature gradient, and one due to the pressure gradient. ${ }^{36}$ The amplitude of this pressure term is controlled by the barodiffusion coefficient $k_{p}$. 
Molecular diffusion in an ideal gas mixture increases as the pressure decreases towards higher altitudes, the expression of $k_{p}$ is known for an ideal binary gas mixture, and turbulent diffusion in usual atmospheric conditions is strong enough to homogenize atmospheric composition up to the homopause. At this level, molecular diffusion dominates and the barodiffusion induces mass separation of the different compounds. Could high-pressure conditions and departure from the ideal gas law induce strongly non-linear behavior of the barodiffusion coefficient ? For such a gradient to be maintained in the near-surface layer of Venus's atmosphere against large-scale and turbulent mixing, the barodiffusion coefficient $k_{p}$ would need to be several orders of magnitude larger than for an ideal gas in the same conditions, which may seem highly unlikely. It is also the case for the previously detailed experiment. ${ }^{30}$ Unfortunately, no measured or theoretical values are yet available for $k_{p}$, neither for the experimental set-up ${ }^{30}$ nor for Venus's deep atmospheric conditions. In the experiments, ${ }^{30,34}$ natural density-driven convection is mentioned as a possible driver, inducing transport of nitrogen-rich lighter parcels upward while $\mathrm{CO}_{2}$-rich heavier parcels would move downward. Additional experimental and theoretical studies are clearly needed to investigate this possibility and to solve this puzzle.

\section{Dynamics of the deep atmosphere of Venus}

To better understand the dynamical state of the different atmospheric layers, as well as the behavior of the PBL near the surface of Venus, the atmospheric circulation was explored using the Laboratoire de Meteorologie Dynamique (LMD) Venus General Circulation Model (GCM). ${ }^{37}$ The variation of the mean molecular mass with pressure in the deep atmosphere was implemented in the computation of the potential temperature within the GCM, though this modification only slightly affects the dynamical state ot the deepest layers. Fitting the observed temperature structure in detail with a radiative transfer model is challenging, because of the sensitivity of the temperature profile to many parameters that are not well known. ${ }^{38}$ However, 
with a fine tuning of these parameters (detailed in the online Methods section), the GCM is able to reproduce the vertical structure of the potential temperature. Therefore, the mean meridional circulation and the turbulent activity diagnosed by the GCM (Fig. 4) can be used to evaluate the dynamical conditions within the atmosphere, including the deepest layer discussed here, despite the large difficulty to get observational constraints for this region.

The deepest layer (below $8 \mathrm{~km}$ ) is close to neutral stability. In the simulation, it is slightly turbulent only near its top, and near the surface with a diurnal convective layer that reaches 1 to $2 \mathrm{~km}$ above the surface around noon local time. This result of the GCM radiative transfer is obtained both when taking into account the composition variation and when composition is uniform. The mean meridional circulation participates in the mixing of the energy through a surface Hadley-type cell roughly 7-km thick. This is similar to the 2-km thick seasonal PBL observed on Titan by the Huygens probe, associated with the mixing by the deepest mean meridional circulation cells. ${ }^{39}$ The hypothetical separation of $\mathrm{N}_{2}$ and $\mathrm{CO}_{2}$ that would explain the VeGa-2 potential temperature profile in the deepest layer needs to occur on timescales shorter than the dynamical overturning of this surface cell $\left(\tau_{d y n}=L / \bar{v}\right.$, where $L \sim 10^{4} \mathrm{~km}$ is the horizontal size of the cell and $\bar{v} \sim 0.05 \mathrm{~m} / \mathrm{s}$ is the mean meridional wind near the surface, yielding $\tau_{d y n} \sim 2 \times 10^{8} \mathrm{~s}$, or $20 \mathrm{Vd}$ ) in order to maintain this vertical gradient in the atmospheric composition, while the layer is close to convective instability. The simulation confirms the very small spatial and temporal variations of the temperature profile, with a diurnal cycle only active near the surface.

\section{Dense gas separation at Venus and beyond}

The unexplained behavior of the $\mathrm{CO}_{2} / \mathrm{N}_{2}$ mixture in the temperature and pressure conditions of the deep atmosphere of Venus needs to be confirmed. First, it illustrates how important it is to go back to Venus to make additional in-situ measurements down to the surface. Second, 
210 further studies are needed, both theoretical and experimental. The compositional gradient de211 duced from our interpretation of the $\mathrm{VeGa}-2$ profile $(5 \mathrm{ppm} / \mathrm{m})$ could be measured in a large experimental tank where Venus' atmospheric conditions can be reproduced. Such a result could trigger interest for theoretical and experimental studies dedicated to other binary mixtures, that could be relevant for the high-pressure atmospheres of giant planets of our own solar system, or for extra-solar planets. 


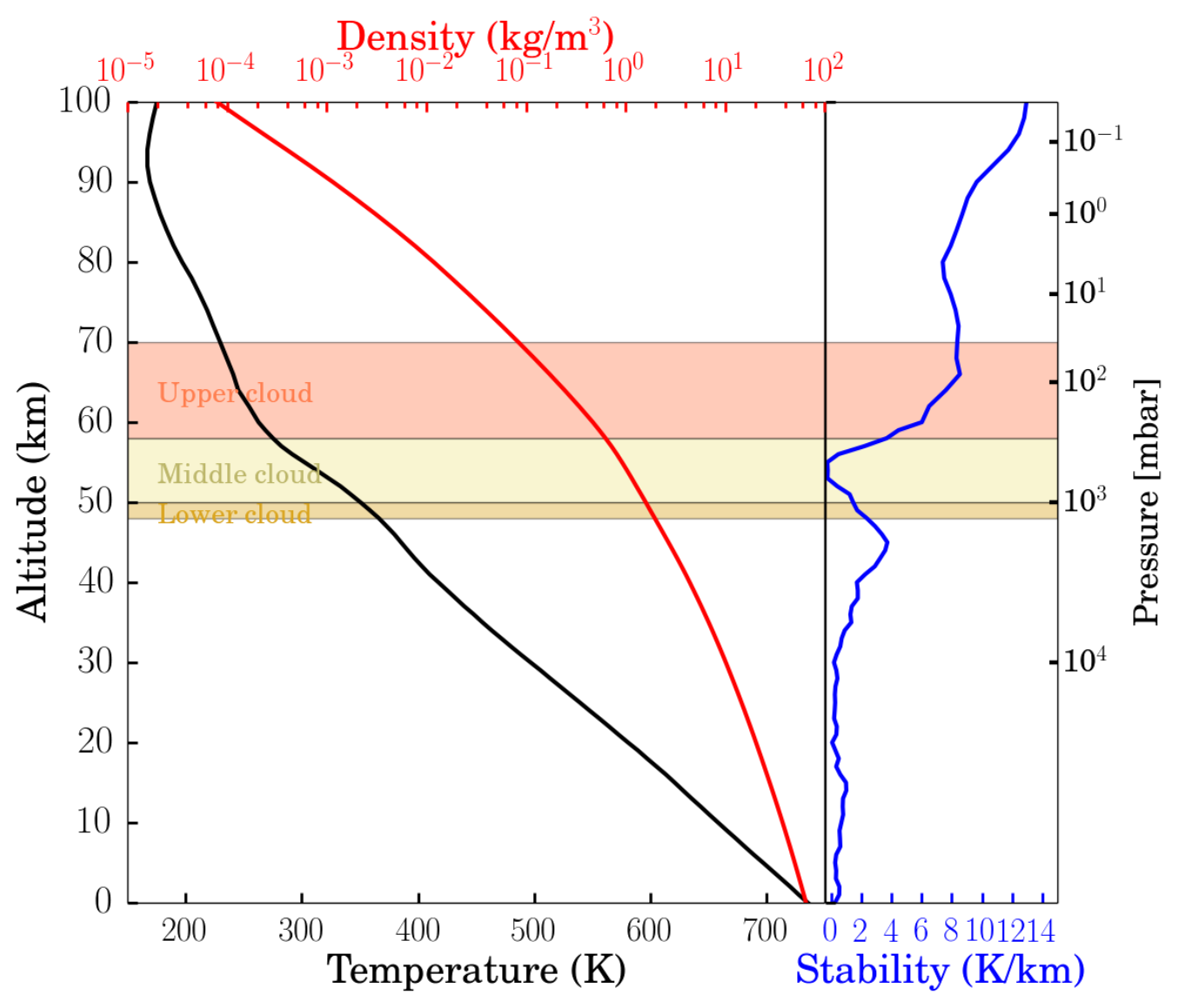

Figure 1 | Vertical structure of the atmosphere of Venus. Vertical profiles, as a function of altitude and pressure, of the temperature, density and static stability (i.e., the difference between the vertical gradient of temperature and the adiabatic lapse rate), from the Venus International Reference Atmosphere model..$^{22}$ Cloud layers are also indicated. 


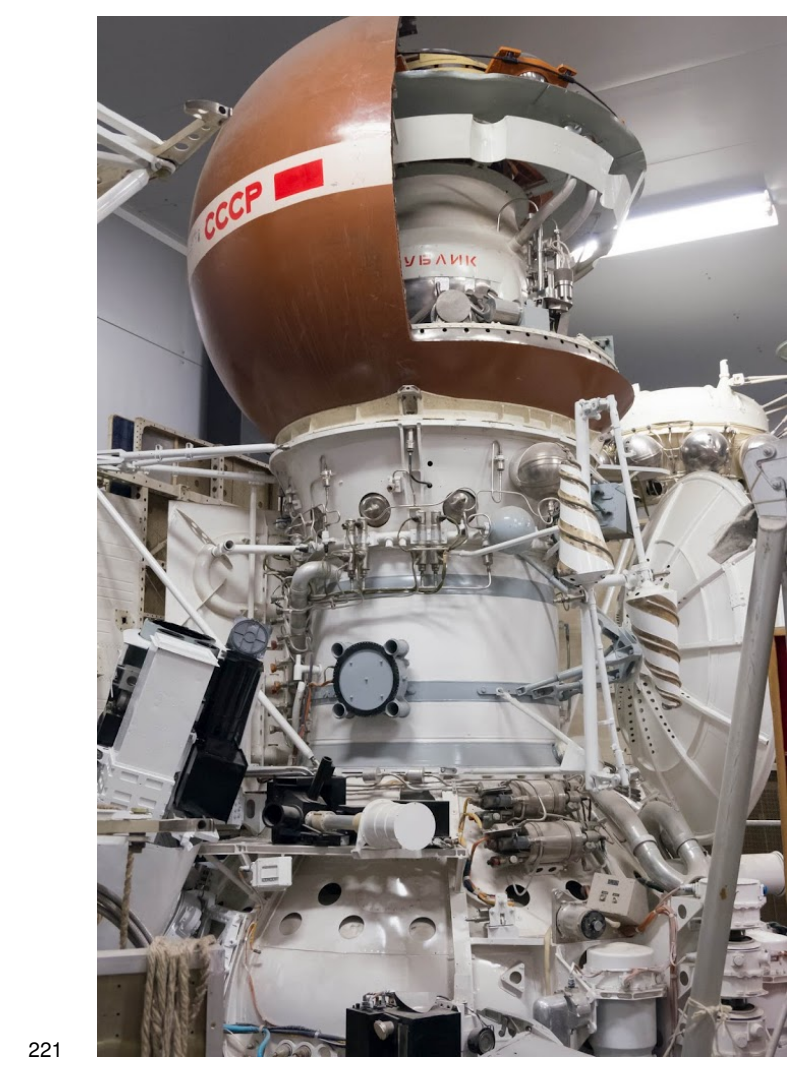

Figure 2 The VeGa-2 spacecraft. Model of the VeGa spacecraft, with the lander visible in the top spherical shell (Lavochkin Museum, near Moscow). Image credits: Lavochkin Association. 


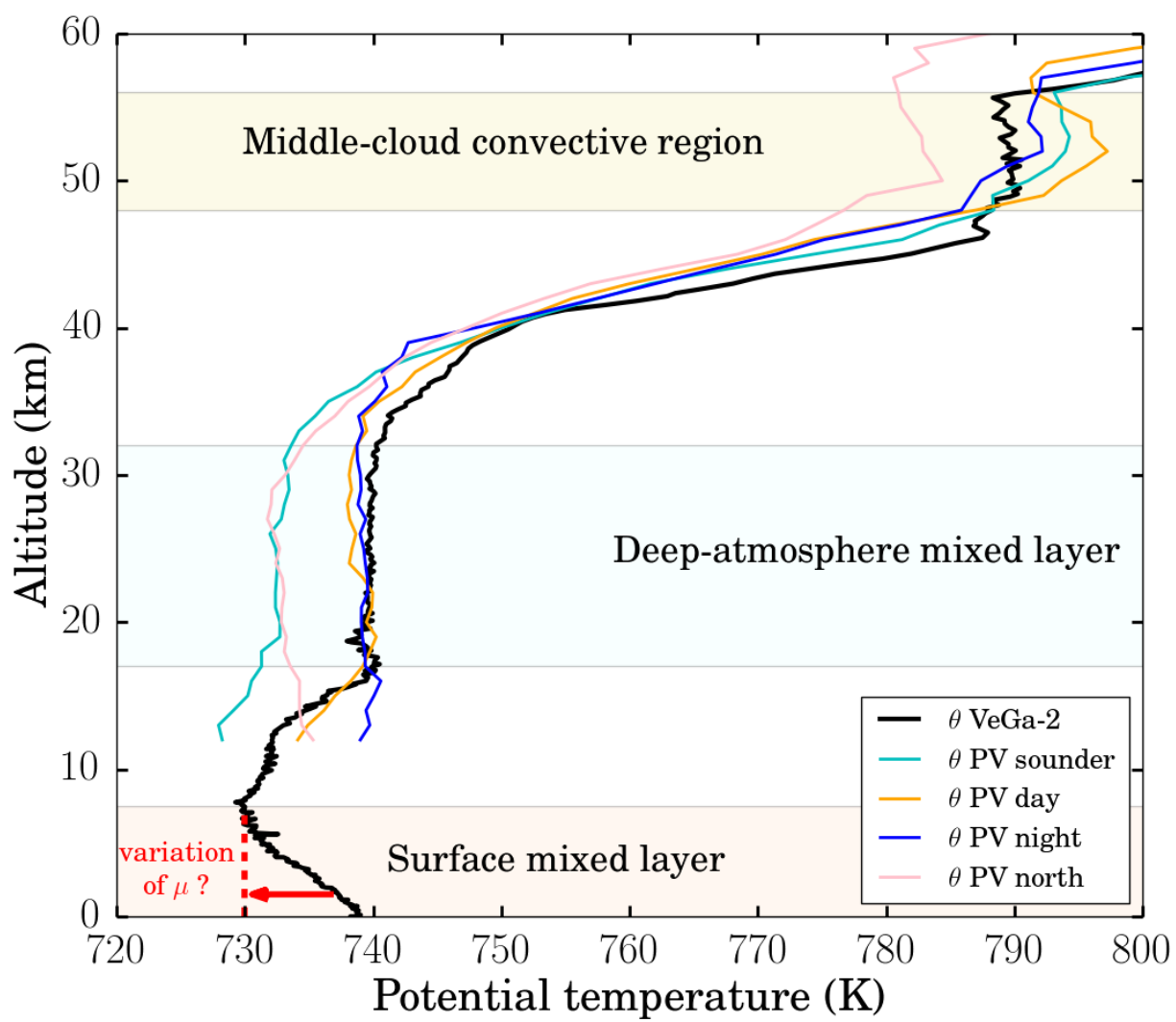

Figure 3 Vertical profile of potential temperature $\theta$ computed from temperatures measured by VeGa-2. Potential temperature is computed using Eq. S10 in the online Methods. VeGa-2 profile shows the convective layer present in the middle and lower clouds (48-56 km altitude), observed in all in-situ and radio-occultation datasets, ${ }^{14,22}$ as well as a deep-atmosphere mixed layer (17-32 km altitude), consistent with the Venus International Reference Atmosphere (VIRA) model ${ }^{22}$ and the Pioneer Venus Sounder, Day and Night probes. ${ }^{16}$ The highly unstable 7-km thick surface layer is also highlighted ( $\mu$ is the mean molecular mass of the atmosphere). 


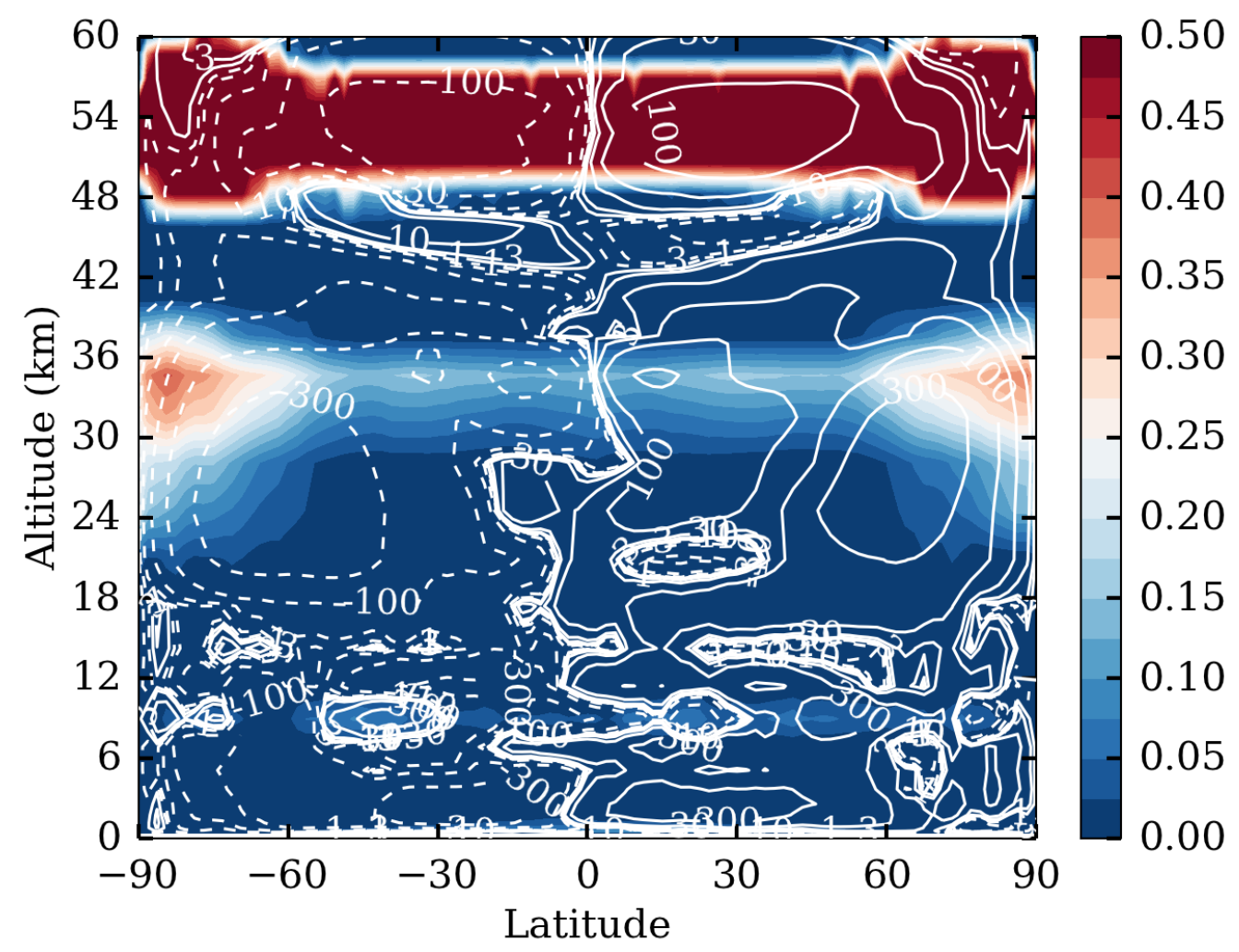

Figure 4 Meridional distributions of the turbulent mixing coefficient and averaged stream

function. The diurnal and zonal average of the turbulent mixing coefficient $K_{z}$ diagnosed in the GCM is shown with colors (unit is $\mathrm{m}^{2} / \mathrm{s}$ ), showing convective regions, while the mean meridional circulation is illustrated by the averaged stream function with the white contours (unit is $10^{9} \mathrm{~kg} / \mathrm{s}$ ). The amplitude of $K_{z}$ reaches more than $10 \mathrm{~m}^{2} / \mathrm{s}$ in the cloud turbulent layer $(48-57 \mathrm{~km})$. 


\section{Box 1}

The stability of an atmospheric region is assessed by moving adiabatically an air parcel along the vertical. For an ideal gas, its temperature follows the adiabatic lapse rate $\left(\frac{d T}{d z}\right)_{a d i a b}=\Gamma=-\frac{g}{c_{p}}$, where $g$ is the gravity and $c_{p}$ is the specific heat capacity at constant pressure. In a well mixed atmosphere (constant molecular mass $\mu$ ), if the parcel rises to a colder environment (or sinks to a warmer environment), it will continue to rise (or sink), becoming buoyant and triggering convective activity. This corresponds to a vertical temperature gradient lower than the adiabatic lapse rate. The stability can then be assessed with the static stability: $S=\frac{d T}{d z}-\Gamma$ : when $S$ is positive, the atmosphere is stable, but when $S$ is negative, convective activity will mix energy and modify the temperature profile until $S=0$.

The potential temperature $\theta$ is defined as the temperature that an air parcel would get after undergoing an adiabatic displacement from its position $(T, p)$ to a reference pressure $p_{\text {ref }}$. The static stability $S$ is equivalent to the vertical gradient of the potential temperature, $\frac{1}{\theta} \frac{d \theta}{d z}$.

When the mean molecular mass is not constant with altitude, to define the buoyancy of a given parcel, the relevant variable is the potential density $\rho_{\theta}$, defined as the density a parcel with the density $\rho(\mu, T, p)$ would have when displaced adiabatically (and with constant composition) to the reference pressure $p_{r e f}, \rho_{\theta}\left(\mu, \theta, p_{\text {ref }}\right)$. In the case of the deep atmosphere of Venus, the stability criterion can be reduced to the usual criterion, but applied to the modified potential temperature $\theta^{\prime}=\theta\left(\mu_{\text {ref }} / \mu\right)$, with $\mu_{\text {ref }}=43.44 \mathrm{~g} / \mathrm{mol}$ a reference value corresponding to $\mathrm{CO}_{2}$ mixed with $3.5 \%$ of $\mathrm{N}_{2}: \frac{1}{\theta^{\prime}} \frac{d \theta^{\prime}}{d z} \geq 0$.

Additional details may be found in the online Methods section. 


\section{References}

1. Crisp, D. \& Titov, D. The thermal balance of the Venus atmosphere. In S. W. Bougher, D. M. Hunten and R. J. Phillips (ed.) Venus II, geology, geophysics, atmosphere, and solar wind environnement, 353-384 (Univ. of Arizona Press, 1997).

2. von Zahn, U., Kumar, S., Niemann, H. \& Prinn, R. Composition of the Venus atmosphere. In D. M. Hunten, L. Colin, T. M. Donahue and V. I. Moroz (ed.) Venus, 299-430 (Univ. of Arizona Press, 1983).

3. Taylor, F. W., Crisp, D. \& Bézard, B. Near-infrared sounding of the lower atmosphere of Venus. In S. W. Bougher, D. M. Hunten and R. J. Phillips (ed.) Venus II, geology, geophysics, atmosphere, and solar wind environnement, 325-351 (Univ. of Arizona Press, 1997).

4. de Bergh, C. et al. The composition of the atmosphere of Venus below 100km altitude: An overview. Planet. \& Space Sci. 54, 1389-1397 (2006).

5. Esposito, L. W., Knollenberg, R. G., Marov, M. I., Toon, O. B. \& Turco, R. P. The clouds and hazes of Venus, 484-564 (Univ. of Arizona Press, 1983).

6. Oertel, D. et al. Infrared spectrometry of Venus from Venera-15 and Venera-16. Adv. Space Res. 5, 25-36 (1985).

7. Moroz, V. I., Linkin, V. M., Matsygorin, I. A., Spaenkuch, D. \& Doehler, W. Venus spacecraft infrared radiance spectra and some aspects of their interpretation. Appl. Opt. 25, 1710-1719 (1986). 
8. Yakovlev, O. I., Matyugov, S. S. \& Gubenko, V. N. Venera-15 and -16 middle atmosphere profiles from radio occultations: Polar and near-polar atmosphere of Venus. Icarus 94, 493-510 (1991).

9. Kliore, A. J. \& Patel, I. R. Vertical structure of the atmosphere of Venus from Pioneer Venus orbiter radio occultations. J. Geophys. Res. 85, 7957-7962 (1980).

10. Taylor, F. W. et al. Structure and meteorology of the middle atmosphere of Venus: Infrared remote sounding from the Pioneer Orbiter. J. Geophys. Res. 85, 7963-8006 (1980).

11. Hinson, D. P. \& Jenkins, J. M. Magellan radio occultation measurements of atmospheric waves on Venus. Icarus 114, 310-327 (1995).

12. Drossart, P. et al. Scientific goals for the observation of Venus by VIRTIS on ESA/Venus Express mission. Planet. \& Space Sci. 55, 1653-1672 (2007).

13. Bertaux, J.-L. et al. SPICAV on Venus Express: Three spectrometers to study the global structure and composition of the Venus atmosphere. Planet. \& Space Sci. 55, 1673-1700 (2007).

14. Tellmann, S., Pätzold, M., Hausler, B., Bird, M. K. \& Tyler, G. L. Structure of the Venus neutral atmosphere as observed by the radio science experiment VeRa on Venus Express. J. Geophys. Res. 114, E00B36 (2009).

15. Keldysh, M. V. Venus exploration with the Venera 9 and Venera 10 spacecraft. Icarus 30, 605-625 (1977).

16. Seiff, A. et al. Measurements of thermal structure and thermal contrasts in the atmosphere of Venus and related dynamical observations - Results from the four Pioneer Venus probes. J. Geophys. Res. 85, 7903-7933 (1980). 
17. Linkin, V. M. et al. Vertical thermal structure in the Venus atmosphere from provisional Vega 2 temperature and pressure data. Sov. Astron. Lett. 12, 40-42 (1986).

18. Linkin, V. M., Blamont, J., Deviatkin, S. I., Ignatova, S. P. \& Kerzhanovich, V. V. Thermal structure of the Venus atmosphere according to measurements with the Vega-2 lander. Kosm. Issled. 25, 659-672 (1987).

19. Bezard, B., de Bergh, C., Crisp, D. \& Maillard, J.-P. The deep atmosphere of Venus revealed by high-resolution nightside spectra. Nature 345, 508-511 (1990).

20. Pollack, J. B. et al. Near-infrared light from Venus' nightside - A spectroscopic analysis. Icarus 103, 1-42 (1993).

21. Meadows, V. S. \& Crisp, D. Ground-based near-infrared observations of the Venus nightside: The thermal structure and water abundance near the surface. J. Geophys. Res. 101, 4595-4622 (1996).

22. Seiff, A., Schofield, J. T., Kliore, A. J. et al. Model of the structure of the atmosphere of Venus from surface to $100 \mathrm{~km}$ altitude. Adv. Space Res. 5, 3-58 (1985).

23. Zasova, L. V., Ignatiev, N. I., Khatuntsev, I. A. \& Linkin, V. Structure of the Venus atmosphere. Planet. \& Space Sci. 55, 1712-1728 (2007).

24. Hoffman, J. H., Oyama, V. I. \& von Zahn, U. Measurement of the Venus lower atmosphere composition - A comparison of results. J. Geophys. Res. 85, 7871-7881 (1980).

25. von Zahn, U. \& Moroz, V. Composition of the Venus atmosphere below $100 \mathrm{~km}$ altitude. Adv. Sp. Res. 5, 173-195 (1985).

26. Avduevskii, V. S. et al. Automatic stations Venera 9 and Venera 10 - Functioning of descent vehicles and measurement of atmospheric parameters. Cosmic Res. 14, 655-666 (1977). 
27. Zasova, L. V., Moroz, V. I., Linkin, V. M., Khatuntsev, I. V. \& Maiorov, B. S. Structure of the Venusian atmosphere from surface up to $100 \mathrm{~km}$. Cosmic Res. 44, 364-383 (2006).

28. Seiff, A. \& the VEGA Baloon Science Team. Further information on structure of the atmosphere of Venus derived from the VEGA Venus Balloon and Lander mission. Adv. Space Res. 7, 323-328 (1987).

29. Gierasch, P. J. et al. The general circulation of the Venus atmosphere: an assessment. In S. W. Bougher, D. M. Hunten and R. J. Phillips (ed.) Venus II, geology, geophysics, atmosphere, and solar wind environnement, 459-500 (Univ. of Arizona Press, 1997).

30. Hendry, D. et al. Exploration of high pressure equilibrium separations of nitrogen and carbon dioxide. J. $\mathrm{CO}_{2}$ Utilization 3-4, 37-43 (2013).

31. Spiga, A., Forget, F., Lewis, S. R. \& Hinson, D. P. Structure and dynamics of the convective boundary layer on Mars as inferred from large-eddy simulations and remote-sensing measurements. Q. J. R. Meteorol. Soc. 136, 414-428 (2010).

32. Read, P. L. et al. Global energy budgets and 'Trenberth diagrams' for the climates of terrestrial and gas giant planets. Quater. J. R. Met. Soc. 142, 703-720 (2016).

33. Wordsworth, R. D. Atmospheric nitrogen evolution on Earth and Venus. Earth and Planet. Sci. Lett. 447, 103-111 (2016).

34. Espanani, R., Miller, A., Busick, A., Hendry, D. \& Jacoby, W. Separation of $\mathrm{N}_{2} / \mathrm{CO}_{2}$ mixture using a continuous high-pressure density-driven separator. J. $\mathrm{CO}_{2}$ Utilization 14, 67-75 (2016). 
35. Span, R. \& Wagner, W. A New Equation of State for Carbon Dioxide Covering the Fluid Region from the Triple-Point Temperature to $1100 \mathrm{~K}$ at Pressures up to $800 \mathrm{MPa}$. J. Phys. Chem. Ref. Data 25, 1509-1596 (1996).

36. Landau, L. D. \& Lifshitz, E. M. Course of Theoretical Physics vol. 6: Fluid mechanics (Pergamon Press, Oxford, UK, 1959).

37. Lebonnois, S., Sugimoto, N. \& Gilli, G. Wave analysis in the atmosphere of Venus below 100-km altitude, simulated by the LMD Venus GCM. Icarus 278, 38-51 (2016).

38. Lebonnois, S., Eymet, V., Lee, C. \& Vatant d'Ollone, J. Analysis of the radiative budget of Venus atmosphere based on infrared Net Exchange Rate formalism. J. Geophys. Res. Planets 120, 1186-1200 (2015).

39. Charnay, B. \& Lebonnois, S. Two boundary layers in Titan's lower troposphere inferred from a climate model. Nature Geosci. 5, 106-109 (2012).

\section{Acknowledgements}

The authors thank Ludmila Zasova for providing the VeGa-2 probe temperature profile, and Josette Bellan for mentioning barodiffusion and useful discussion on this phenomenon. S.L. acknowledges the support of the Centre National d'Etudes Spatiales. G.S. acknowledges the support of the Keck Institute for Space Studies under the project "Techniques and technologies for investigating the interior structure of Venus". 
362

363

364

\section{Methods}

\section{Stability and potential temperature}

The stability of an air parcel undergoing an adiabatic displacement in situations where $\mu$ and/or $c_{p}$ may depend on altitude, pressure or temperature is detailed in the following study. The notations used are as follows: $R$ is the universal gas constant $\left(R=8.3144621 \mathrm{~J} \mathrm{~mol}^{-1} \mathrm{~K}^{-1}\right), \mu$ is the mean molecular mass, $p$ is the pressure, $\rho$ is the density, $v=1 / \rho$ is the specific volume, $T$ is the temperature, $c_{p}$ and $c_{v}$ are the specific heat capacities at constant pressure and constant volume, $\lambda=c_{p} / c_{v}$ and $\kappa=R /\left(\mu c_{p}\right)$.

Initial equations. The basic equations for this study are:

- the specific heat relations

(Eq. S1)

$$
d U=c_{v} d T
$$

(Eq. S2)

$$
\frac{R}{\mu}=c_{p}-c_{v}
$$

which yields

$$
\kappa=1-\frac{1}{\lambda}
$$

- the first law of thermodynamics for adiabatic displacement

(Eq. S3)

$$
d U=-p d v
$$

- the equation of state for an ideal gas

(Eq. S4)

$$
\rho=\frac{\mu p}{R T}
$$


Note that in the case of the deep atmosphere of Venus, the ideal gas law is only an approximation, but with an error on density less than $0.8 \%$ (Table S1). ${ }^{19,35}$

- the hydrostatic balance

(Eq. S5)

$$
d p=-\rho g d z
$$

When $\mu$ is constant in the atmosphere. In the cases where $\mu$ is constant in the atmosphere, Eq. S4 can be written as:

$$
p v=\frac{R}{\mu} T
$$

Differentiating this equation yields

(Eq. S6)

$$
p d v+v d p=\frac{R}{\mu} d T
$$

From Eqs. S1 and S3, we get

$$
p d v=-c_{v} d T
$$

Together with Eq. S2, Eq. S6 becomes

$$
v d p=c_{p} d T
$$

Using Eq. S4 again, this yields

(Eq. S7)

$$
\frac{R}{\mu} \frac{d p}{p}=c_{p} \frac{d T}{T}
$$

The potential temperature $\theta$ is defined as the temperature that an air parcel would get after undergoing an adiabatic displacement to a reference pressure $p_{r e f}$. Its expression is obtained by integrating this adiabatic displacement from $(T, p)$ to $\left(\theta, p_{r e f}\right)$. When $c_{p}$ is constant, Eq. S7 yields the usual expression 
(Eq. S8)

$$
\theta=T\left(\frac{p_{r e f}}{p}\right)^{\kappa}
$$

When $c_{p}$ depends on the temperature, the integration is not direct. Using the expression (Eq. S9)

$$
c_{p}=c_{p 0}\left(\frac{T}{T_{0}}\right)^{\nu}
$$

(with $c_{p 0}=1000 \mathrm{~J} / \mathrm{kg} / \mathrm{K}, T_{0}=460 \mathrm{~K}$ and $\nu=0.35$ for Venus' atmosphere), ${ }^{35,40,41}$ it can be demonstrated $^{40}$ that the new expression for $\theta$ is:

(Eq. S10)

$$
\theta^{\nu}=T^{\nu}+\nu T_{0}^{\nu} \ln \left(\frac{p_{r e f}}{p}\right)^{\kappa_{0}}
$$

with $\kappa_{0}=R /\left(\mu c_{p 0}\right)$.

Using Eqs. S4, S5 and S7 yields

$$
-\frac{g d z}{T}=c_{p} \frac{d T}{T}
$$

which gives the adiabatic lapse rate (valid even for variable $c_{p}$ )

(Eq. S11)

$$
\left(\frac{d T}{d z}\right)_{a d i a b}=\Gamma=-\frac{g}{c_{p}}
$$

When $\mu$ depends on altitude, pressure or temperature. The stability criterion is established as follows. ${ }^{42,43}$ Consider a parcel that is displaced adiabatically on an elemental distance $d z, q *$ refers to the variable $q$ in the parcel.

Eq. S4 can be written as

$$
p * \mu *=\rho * R T *
$$

Taking the logarithm then differentiating along the vertical axis ( $\mu *$ is constant because the composition of the parcel does not change) yields

$$
\frac{1}{p *} \frac{d p *}{d z}=\frac{1}{\rho *} \frac{d \rho *}{d z}+\frac{1}{T *} \frac{d T *}{d z}
$$


${ }_{411} \quad$ Using Eq. S7 applied to the parcel and $p=p *$ yields

$412 \quad$ (Eq. S12)

$$
\frac{1}{\rho *} \frac{d \rho *}{d z}=\frac{1}{p} \frac{d p}{d z}(1-\kappa *)
$$

413

with $\kappa *=R /\left(\mu * c_{p}\right)$.

For the background gas, Eq. S4 can be written as:

$$
\rho=\frac{\mu p}{R T}
$$

Taking the logarithm then differentiating along the vertical axis yields

(Eq. S13)

$$
\frac{1}{\rho} \frac{d \rho}{d z}=\frac{1}{\mu} \frac{d \mu}{d z}+\frac{1}{p} \frac{d p}{d z}-\frac{1}{T} \frac{d T}{d z}
$$

The stability criterion is

(Eq. S14)

$$
\frac{1}{\rho *} \frac{d \rho *}{d z}>\frac{1}{\rho} \frac{d \rho}{d z}
$$

Eqs. S12 and S13 yield

(Eq. S15)

$$
\frac{1}{\mu} \frac{d \mu}{d z}-\frac{1}{T} \frac{d T}{d z}+\frac{\kappa *}{p} \frac{d p}{d z}<0
$$

Applying this stability criterion, the adiabatic lapse rate is obtained when neutral for stability:

(Eq. S16)

$$
\frac{1}{\mu} \frac{d \mu}{d z}-\frac{1}{T} \frac{d T}{d z}+\frac{\kappa *}{p} \frac{d p}{d z}=0
$$

Using Eqs. S4, S5 and the fact that $\kappa / \kappa *$ tends to 1 for an elemental displacement, this can be written as

(Eq. S17)

$$
\left(\frac{d T}{d z}\right)_{a d i a b}=\Gamma=\frac{T}{\mu} \frac{d \mu}{d z}-\frac{g}{c_{p}}
$$


which is valid even for variable $c_{p}$.

To define the buoyancy of a given parcel, the relevant variable is the potential density $\rho_{\theta}$, defined as the density a parcel with the density $\rho(\mu, T, p)$ would have when displaced adiabatically (and with constant composition) to the reference pressure $p_{r e f}, \rho_{\theta}\left(\mu, \theta, p_{r e f}\right)$. Using the ideal gas law (Eq. S4), the potential density is

(Eq. S18)

$$
\rho_{\theta}=\frac{\mu p_{r e f}}{R \theta}=\frac{\mu_{r e f} p_{r e f}}{R \theta^{\prime}}
$$

with the modified potential temperature $\theta^{\prime}$ defined by

(Eq. S19)

$$
\theta^{\prime}=\theta\left(\mu_{r e f} / \mu\right)
$$

Due to the variation of $\mu$ with altitude and the dependence of $\theta$ on $\mu$, it is not correct to reduce the stability criterion (Eq. S16) to the usual criterion, i.e., the direct comparison of the potential density between two atmospheric levels. ${ }^{44}$

(Eq. S20)

$$
\frac{1}{\rho_{\theta}} \frac{d \rho_{\theta}}{d z}=\frac{1}{\mu} \frac{d \mu}{d z}-\left(\frac{1}{\theta} \frac{\partial \theta}{\partial z}\right)_{\mu}-\left(\frac{1}{\theta} \frac{\partial \theta}{\partial \mu}\right)_{z} \frac{d \mu}{d z}
$$

For an elemental displacement, the definition of $\theta$ yields

(Eq. S21)

$$
\left(\frac{1}{\theta} \frac{\partial \theta}{\partial z}\right)_{\mu}=\frac{1}{T} \frac{d T}{d z}-\frac{\kappa *}{p} \frac{d p}{d z}
$$

which can be inserted in Eq. S20 to give

(Eq. S22)

$$
\frac{1}{\rho_{\theta}} \frac{d \rho_{\theta}}{d z}=\frac{1}{\mu} \frac{d \mu}{d z}-\frac{1}{T} \frac{d T}{d z}+\frac{\kappa *}{p} \frac{d p}{d z}-\left(\frac{1}{\theta} \frac{\partial \theta}{\partial \mu}\right)_{z} \frac{d \mu}{d z}
$$

Eq. S22 shows that $d \rho_{\theta} / d z=0$ (or $d \theta^{\prime} / d z=0$ ) is not equivalent to the stability criterion (Eq. S16), unless the last term of the right side is negligible against the first. 
However, in the case of the deep atmosphere of Venus, the vertical profile of $\theta(\mu)$ is very close (difference less than $0.15 \mathrm{~K}$ everywhere) to the profile of $\theta\left(\mu_{r e f}\right)$, with $\mu_{r e f}=43.44 \mathrm{~g} / \mathrm{mol}$ a reference value corresponding to $\mathrm{CO}_{2}$ mixed with $3.5 \%$ of $\mathrm{N}_{2}$. This yields $(\mu / \theta)(\partial \theta / \partial \mu) \sim$ $(43.44 / 735) \times(0.15 / 0.56) \sim 0.016$, much smaller than 1 . It is therefore a good approximation to consider that the definition of the potential temperature $\theta$ is not dependent on the initial mean molecular mass of the air parcel, i.e., $\partial \theta / \partial \mu=0$ at any given level. In this case, the stability criterion is equivalent to the usual criterion applied to the modified potential temperature $\theta^{\prime}$ :

(Eq. S23)

$$
\frac{1}{\theta^{\prime}} \frac{d \theta^{\prime}}{d z}=0 .
$$

\section{Radiative transfer details}

In the GCM used for our study, the temperature structure is modeled using a full radiative transfer model. In the infrared range, net exchange rate (NER) formalism is used ${ }^{38,45}$ based on up-to-date gas opacities including collision-induced absorption from $\mathrm{CO}_{2}$ dimers ${ }^{46}$, and the most recent cloud model deduced from Venus-Express datasets ${ }^{47}$. In the solar range, vertical profiles of the solar fluxes computed using this new cloud model are used, depending on lati-

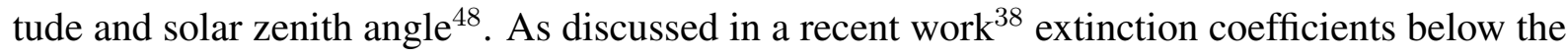
clouds in windows located between 3 and 7 microns play a key role in shaping the deep atmosphere temperature profile. The solar heating profile below the clouds is also crucial, though it is poorly constrained by available data.

Globally averaged 1-dimensional simulations were performed to assess the sensitivity to crucial hypotheses in the radiative transfer calculation. Different solar heating rate models were used $^{48-50}$ (Fig. S1a). The composition of the lower haze particles, located between the cloud base $(48 \mathrm{~km})$ and $30 \mathrm{~km}$ and observed by the probe nephelometers ${ }^{51}$, is not established, so their optical properties are not well constrained. The absorption of the solar flux in this region is 
therefore subject to uncertainty. An increased solar absorption (by a factor 3 ) in this region in the H15 profile ${ }^{48}$ (Fig. S1) provides the best fit to the VIRA and Vega-2 temperature profiles. In the infrared, some additional extinction is needed below the clouds in the 3 to 7 microns wavelength range to fit the temperature profile in the stable region below the clouds ${ }^{38}$. The lower haze, which is not taken into account in the reference NER computations, can contribute to this small additional continuum. The impact of several hypotheses on this additional opacity is illustrated in Fig. S1b. The best fit to the VIRA and Vega-2 temperature profiles is obtained with an additional extinction of $1.3 \times 10^{-6} \mathrm{~cm}^{-1}$ amagat ${ }^{-2}$ in the lower haze region (30-48 km), and of $4 \times 10^{-7} \mathrm{~cm}^{-1}$ amagat $^{-2}$ in the region between 30 and $16 \mathrm{~km}$, where a transition from instability to stability against convection is observed in the Vega-2 profile, but also in the Pioneer Venus Sounder, Day and Night probes at similar altitudes (15 to $20 \mathrm{~km})^{19}$.

\section{References}

40. Lebonnois, S. et al. Superrotation of Venus' atmosphere analysed with a full General Circulation Model. J. Geophys. Res. 115, E06006 (2010).

41. Poling, B., Prausnitz, J. \& O’Connell, J. The properties of gases and liquids (McGraw-Hill, 2001).

42. Ledoux, P. Stellar models with convection and with discontinuity of the mean molecular weight. Astrophys. J. 105, 305-321 (1947).

43. Hess, S. L. Static stability and thermal wind in an atmosphere of variable composition: Applications to Mars. J. Geophys. Res. 84, 2969-2973 (1979).

44. Pierrehumbert, R. T. Principles of Planetary Climate (Cambridge Univ. Press, Cambridge, UK, 2010). 
45. Eymet, V. et al. Net-exchange parameterization of the thermal infrared radiative transfer in Venus' atmosphere. J. Geophys. Res. 114, E11008 (2009).

46. Stefani, S., Piccioni, G., Snels, M., Grassi, D. \& Adriani, A. Experimental $\mathrm{CO}_{2}$ absorption coefficients at high pressure and high temperature. J. of Quantit. Spec. and Rad. Transfer 117, 21-28 (2013).

47. Haus, R., Kappel, D. \& Arnold, G. Atmospheric thermal structure and cloud features in the southern hemisphere of Venus as retrieved from VIRTIS/VEX radiation measurements. Icarus 232, 232-248 (2014).

48. Haus, R., Kappel, D. \& Arnold, G. Radiative heating and cooling in the middle and lower atmosphere of Venus and responses to atmospheric and spectroscopic parameter variations. Planet. \& Space Sci. 117, 262-294 (2015).

49. Crisp, D. Radiative forcing of the Venus mesosphere. I - Solar fluxes and heating rates. Icarus 67, 484-514 (1986).

50. Lee, C. \& Richardson, M. I. A Discrete Ordinate, Multiple Scattering, Radiative Transfer Model of the Venus Atmosphere from 0.1 to $260 \mu$ m. J. Atm. Sci. 68, 1323-1339 (2011).

51. Knollenberg, R. G. et al. The clouds of Venus: A synthesis report. J. Geophys. Res. 85, 8059-8081 (1980).

\section{Data and code availability}

The VeGa-2 temperature profile was provided kindly provided by Ludmila Zasova. It is available from the corresponding author upon request.

The LMD Venus GCM used in this study is developed in the corresponding author's team. It is also available upon request. 


\section{${ }_{512}$ Author contributions}

513 Both authors contributed equally to the manuscript.

\section{${ }_{514}$ Additional information}

Methods section and Supplementary Information are available in the online version of the paper.

Correspondence and requests for materials should be addressed to S.L.

\section{Competing financial interests}

The authors declare no competing financial interests. 